\title{
Effects of the stress of marathon running on implicit and explicit memory
}

\author{
Teal S. Eich and Janet Metcalfe \\ Columbia University, New York, New York
}

\begin{abstract}
We tested the idea that real-world situations, such as the highly strenuous exercise involved in marathon running, that impose extreme physical demands on an individual may result in neurohormonal changes that alter the functioning of memory. Marathon runners were given implicit and explicit memory tasks before or immediately after they completed a marathon. Runners tested immediately upon completing the marathon showed impairment in the explicit memory task but enhancement in the implicit memory task. This postmarathon impairment in explicit memory is similar to that seen with amnesic patients with organic brain damage. However, no previous studies have shown a simultaneous enhancement in the implicit memory task, as shown by the marathon runners in the present study. This study indicates that human memory functioning can be dynamically altered by such activities as marathon running, in which hundreds of thousands of healthy normal individuals routinely partake.
\end{abstract}

With the increasing understanding of the selective effects of neuromodulators, including stress hormones, that vary with the vicissitudes of daily living, comes the need to investigate human memory under the kinds of naturalistic conditions that may give rise to changes in the balance of these neuromodulators. Patterns of memory responses exhibited in the laboratory may or may not generalize to real-world situations in which neuromodulator levels are altered. Memory dissociations have often been found with patient populations with focal brain damage. But such lesions are not the only, or even the most common, situation in which different memory systems or processes may be selectively engaged. The possibility exists that the fluctuations in neuromodulators obtained under real-world conditions could produce in normal individuals memory dissociations similar to those seen in patient populations; an understanding of such dissociations may be crucial for understanding the dynamics of human memory in the wild.

Exercise is an interesting (and pervasive) example of a behavior that has an influence on neuromodulators affecting memory. The emotional, cognitive, and physiological effects of moderate levels of aerobic exercise, which we would not consider stressful, have been well documented. These include beneficial effects on mood (Byrne \& Byrne, 1993), cognitive speed, auditory and visual attention (Angevaren, Aufdemkampe, Verhaar, Aleman, \& Vanhees, 2008), and neurogenesis in the dentate gyrus of the hippocampus, a brain structure implicated in memory (Pereira et al., 2007).

But what about the effects of heavy, strenuous exercise? Research into the physiological effects of marathon running, the event on which we focus in the present study, suggests the possibility of maladaptive consequences.
Dehydration, gastrointestinal bleeding, muscle damage, immune suppression, and even sudden cardiac death have all been reported (see Uchakin, Gotovtseva, \& Stray-Gundersen, 2003). Marathon running also greatly increases production of cortisol and norepinephrine, hormones that are elevated by physiological and emotional stress. Marathon runners' cortisol levels have been documented (Cook, Ng, Read, Harris, \& Riad-Fahmy, 1987) to rise fourfold above the highest levels induced by the most common laboratory-based stress task, the Trier Social Stress Task (Kirschbaum, Pirke, \& Hellhammer, 1993). Indeed, cortisol levels recorded $30 \mathrm{~min}$ after completion of a marathon rival those reported in military training and interrogation (Taylor et al., 2007), rape victims being treated acutely (Resnick, Yehuda, Pitman, \& Foy, 1995), severe burn injury patients (Norbury, Herndon, Branski, Chinkes, \& Jeschke, 2008), and first-time parachute jumpers (Aloe et al., 1994). Other neurotransmitters, such as norepinephrine, are similarly elevated in marathon running (Demers, Harrison, Halbert, \& Santen, 1981). Leading experts (Sapolsky, 2004, p. 104) have concluded that marathon running is one of the most stressful activities in which normal, neurologically intact humans engage. The affective valence of marathon running is, of course, quite different from that of the traumatic situations referenced above. However, the physiological response is on a par with these events. No studies to date have examined the effects of marathon running on memory function, despite the opportunity that marathon running offers to ethically study the memorial impact of extremely high levels of stress hormones.

Here, we addressed the effects of marathon running on implicit and explicit memory tasks. We chose these tasks

T. S. Eich, tse4@columbia.edu 
because they previously have been shown to dissociate with certain brain-damaged patients (see Graf \& Schacter, 1985). Patients who have sustained hippocampal damage show impairments in what Graf and Schacter called explicit memories, memories elicited by questions such as "what happened an hour ago?" or "what were the items on the list you just read?" If shown a list of words and told to remember them, patients may be unable to recall any of them. Despite this deficit, these patients show intact implicit memory. They exhibit performance as good as that of nonamnesics for the words on the list in an implicit memory task where a few letters of the words are given and the patient is asked to complete the words.

Other research has linked elevated cortisol levels to deficits in explicit memory. In these studies, high doses of cortisol alone have been administered, either orally or intravenously, in the form of methylprednisolone, a synthetic glucocorticoid. The results showed decreases in verbal declarative memory and word recall (e.g., de Quervain, Roozendaal, Nitsch, McGaugh, \& Hock, 2000; Newcomer et al., 1999). One explanation for these findings is that cortisol's affinity within the hippocampus for binding to glucocorticoid receptors may have resulted in a reversible hippocampal dysfunction (de Kloet, Vreugdenhil, Oitzl, \& Joëls, 1998). Thus, the cortisol studies and amnesia studies may be related, with memory deficits in both being attributable to hippocampal impairment - permanent in one case, reversible in the other. On the basis of these results, we hypothesized that marathon runners immediately after the race, when cortisol levels are at their highest levels, but not several days before the race, when cortisol levels are close to normal, would show deficits in an explicit memory task. Marathon running, however, results in many changes other than increased cortisol levels that could contribute to impairments in explicit memory.

What should happen to other kinds of memory is less clear. Demers et al. (1981) reported that epinephrine and norepinphrine are greatly increased during and after a marathon. Increases in such neurotransmitters have been shown to result in enhancements in emotional responses, fear, and appetitive conditioning (Cahill \& Alkire, 2003; LeDoux, 1996; McGaugh, 2004). Given that marathon running augments stress hormones, other than cortisol, that might enhance memory performance, it seemed possible that performance on an implicit memory task might either be spared, as with amnesic patients, or indeed might be enhanced. Evolutionarily, it seems plausible that a stressor not only might have inhibiting effects, but also might serve to selectively enhance certain functions.

We used the same implicit word-stem completion and explicit cued recall tasks and instructions used by Graf and Schacter (1985) that showed the classic dissociation of memory function in amnesic patients. Our hypothesis was that when marathon runners had just undergone the stress of running a marathon as compared with several days earlier, their performance on the explicit cued recall task would be impaired. At the same time, we predicted that their performance on the implicit stem-completion task would be spared or might even be enhanced.

\section{METHOD}

\section{Participants}

The 261 participants included in the sample were between the ages of 18 and 65 and were verified by bib number to have completed either the New York City Marathon or the Boston Marathon during the week in which we tested them. Twenty-two additional people who were tested were excluded without being scored because they were observed by the experimenter to have cheated or because their native language was not English. The control condition participants were 120 marathon runners ( 55 men, 65 women; mean age = 36.56 years) recruited and tested at an event 1-3 days before the marathon that they ran. Sixty-eight were tested between noon and 5 p.m.; 52 were tested between 6 p.m. and 9 p.m. Using marathon runners who were confirmed to have completed the race as a control group helped to ensure that observed differences in memory were related to the effects of having just run the race, rather than to personality, fitness, socioeconomic status, or the myriad of other factors that might differentiate people who run marathons from people who do not. We could not rigorously control all possible factors. For example, although it was our subjective impression that the overwhelming majority of people in both groups agreed to participate, a few declined. The reasons given were that they were meeting friends or going to eat; in the marathon group, a few declined because they were tired. However, few in either group declined. Participants in the marathon group $(n=141 ; 86$ men, 55 women; mean age $=$ 36.21 years) were recruited and tested between noon and 5 p.m. at the race's designated reception area, approximately $30 \mathrm{~min}$ after completing the marathon.

All participants, who were unpaid volunteers, gave informed consent before they began the study, a process that conformed to APA guidelines and that was approved by the Columbia University Institutional Review Board. Participants also gave permission to have their times verified.

The amount of noise and celebration was approximately the same at the prerace convention areas and at the postrace meeting areas where we tested. It was decided not to recruit participants at the Los Angeles Marathon, for example, because loud music, which could pose an external distraction, was played at the finish of that race. The New York City Marathon and the Boston Marathon do not have this problem.

\section{Materials}

The materials consisted of 39 words and their 3-letter word stems, chosen from Graf and Williams's (1987) normed word pool. Words consisted of nouns, verbs, and adjectives (range, 4-7 letters; $M=5.6$ ). The materials were randomized individually for each participant: Each item was assigned randomly to be a studied item tested in the explicit condition, a studied item tested in the implicit condition, or an unstudied item tested in the baseline implicit condition. The computer randomly ordered words from the base set of 39 into each condition and printed individual booklets for each participant. To hand score the data, we used individual scoring keys computer-generated for each participant. A grid number allowed the scoring keys to be collated with the test booklets.

\section{Procedure}

After granting informed consent, participants were given the booklet containing the study materials. On the first page was the study event. Participants rated 26 words (chosen at random from the pool of 39) for pleasantness on a 5-point scale. Text on the second page informed participants that each of the cues on the subsequent page would be the beginning of an English word. Their task would be to write a few letters to make each cue into any English word, but they were instructed to write down the first one that came to mind. The next page, which presented the implicit word-stem completion task, contained word stems from 13 words that were previously rated for pleasantness as well as stems from 13 unrated (baseline) words, randomly intermixed. Following completion of the implicit memory task, participants were given instructions for the explicit 
memory cued recall task. They were told that each of the cues on the subsequent page was the beginning of a word for which they had provided a pleasantness rating earlier. They were instructed to try to complete each cue with the word from the pleasantness-rating list - that is, to explicitly remember the previously rated words. They were also instructed not to look back at the list. After reading these instructions, they turned to the last page, which contained the 13 remaining three-letter word stems presented in a random order, all of which they had previously rated for pleasantness. Given the cues, they then attempted to recall the words. Instructions for both tasks were modeled after those developed by Graf and Schacter (1985) to test implicit and explicit memory in amnesic patients. The study was self-paced and took participants an average of $10 \mathrm{~min}$ to complete. The time between study and both memory tasks was marginal.

The implicit task always preceded the explicit task. This fixed task order has been implemented in the majority of published experiments in which these memory measures are manipulated as withinsubjects factors (see Bäckman et al., 1997; Graf \& Mandler, 1984; Graf \& Schacter, 1985). Indeed, in one of the few published studies in which the order of tasks was counterbalanced, problematic order effects affecting the implicit task were reported when the explicit test came first (Kihlstrom, Schacter, Cork, Hurt, \& Behr, 1990). This is because, after performing the explicit memory task, participants are no longer naive to the fact that they are being tested for memory for the words that they had rated for pleasantness. Until the explicit task is given, however, the chance is low that the participants know that the words that they rated are related to the word-stem completion task. Although it does not guarantee that an explicit strategy is not used in the implicit task, maintaining a fixed order, in which the implicit task precedes the explicit task, helps to ensure the implicitness of the implicit task.

\section{RESULTS}

As Figure 1 shows, the proportion of words correctly recalled from the previously studied list on the explicit memory (cued recall) task was lower for the marathon group than for the control group, as predicted. However, on the implicit memory (word-stem completion) task, the marathon group performed better than the control group. The implicit memory score was a priming measure, computed as the difference between the proportions of correctly completed presented and unpresented (baseline) words (i.e., a correctly completed stem from our original list, despite the word's not having been presented at encoding). A repeated measures ANOVA revealed an interaction between group (marathon vs. control) and memory task (implicit vs. explicit) $\left[F(1,259)=16.73, M S_{\mathrm{e}}=5.69\right.$, $\left.p<.001, \eta_{\mathrm{p}}^{2}=.06\right]$. Post hoc tests confirmed that performance on the explicit memory task was worse for the marathon group than for the control group $[t(259)=2.05$, $p=.04]$ and that performance was significantly better for the marathon group, as compared with the control group, on the implicit memory task $[t(259)=-3.38, p=.001]$. Baseline implicit memory performance was the same for the control $(m=.06)$ and marathon $(m=.06)$ groups $[t(259)=1.45, p=.15$, n.s. $]$.

Control group participants averaged slower running times than did marathon group participants $[t(259)=$ $6.62, p<.001]$. We therefore performed two ANCOVAs, the first with implicit score as the dependent variable, group as the fixed factor, and running time as the covariate. There was no effect of running time on implicit memory $[B=-0.12$ scale units $/ \mathrm{h}, S E=0.24 ; t(259)=$ $-0.63, p=.53$, n.s.]. When controlled for time, the group effect was still significant $\left[F(1,258)=8.32, M S_{\mathrm{e}}=6.27\right.$, $p=.004]$. We performed the analogous ANCOVA with the explicit scores. Running time, here, had a significant effect $[B=-0.66$ scale units $/ \mathrm{h}, S E=0.24 ; t(259)=$ $-2.68, p=.008]$. The effect of group, controlled for running time $\left[F(1,258)=8.62, M S_{\mathrm{e}}=6.65, p=.004\right]$, was slightly stronger than before, indicating that the difference in running times across groups had been partially masking the negative effects of marathon running on the explicit memory task.

\section{Additional Analysis}

The interaction persisted when we analyzed the Boston participants $\left[F(1,110)=4.53, M S_{\mathrm{e}}=5.82, p=.035\right.$, $\left.\eta_{\mathrm{p}}^{2}=.04\right]$ and the New York participants $[F(1,147)=$ $\left.6.28, M S_{\mathrm{e}}=5.39, p=.01, \eta_{\mathrm{p}}^{2}=.04\right]$ separately. The interaction remained significant for men alone $[F(1,139)=$

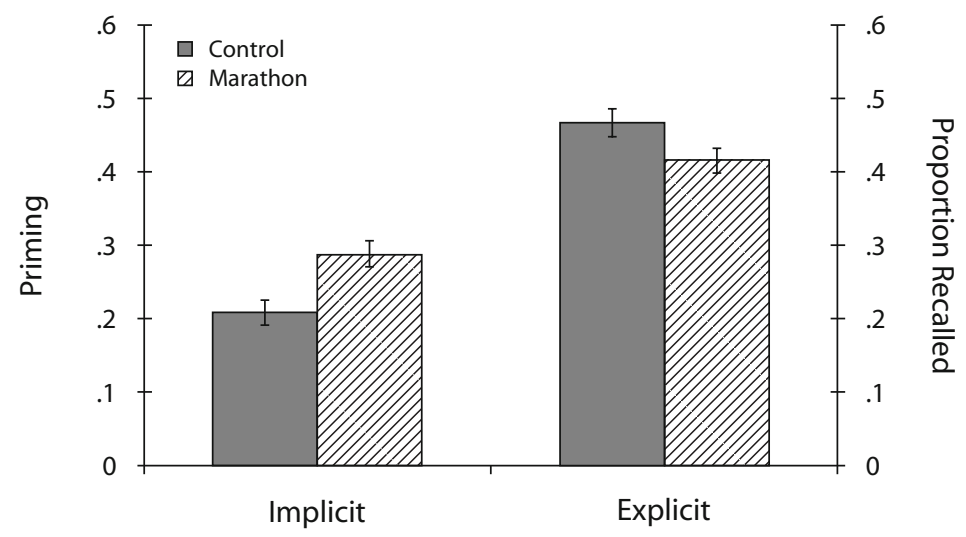

Figure 1. Performance on the implicit and explicit memory tasks. The left bars give the difference between the control (premarathon) and marathon (postmarathon) groups on the implicit memory word-stem completion task. The right bars give control group and marathon group performance on the explicit cued recall task. Priming is the difference in the proportions of correctly completed presented and unpresented (baseline) words. 
6.27, $\left.M S_{\mathrm{e}}=5.51, p=.014, \eta_{\mathrm{p}}^{2}=.04\right]$ and for women alone $\left[F(1,118)=8.63, M S_{\mathrm{e}}=5.91, p=.004, \eta_{\mathrm{p}}^{2}=.07\right]$. Although there was no difference in age between the two groups, we nevertheless ran two separate ANCOVAS, with group as the fixed factor and age as the covariate.

Neither implicit performance $[B=0.02$ scale units/ year, $S E=0.02, T=1.03, p=.31$, n.s.; $F(1,258)=$ $\left.11.57, M S_{\mathrm{e}}=6.26, p=.001\right]$ nor explicit performance $[B=-0.007$ scale units/year, $S E=0.19, T=-0.35, p=$ .73 , n.s.; $\left.F(1,258)=4.21, M S_{\mathrm{e}}=6.83, p=.04\right]$ showed a group effect of age. Finally, 52 control participants were recruited at a race-related party that took place after 5 p.m. We therefore did a subsequent analysis eliminating these participants and using only control and marathon group participants who were controlled for time of day. As before, the interaction between group (marathon vs. control) and memory task (implicit vs. explicit) was significant $\left[F(1,207)=20.24, M S_{\mathrm{e}}=5.64, p<.001, \eta_{\mathrm{p}}^{2}=.09\right]$.

\section{DISCUSSION}

These data revealed a double dissociation of the effects of marathon running on an implicit and an explicit memory task. We interpret the results as suggesting that complex neuromodulation associated with extreme stress enhances some brain systems and inhibits others; however, other explanations could be raised. ${ }^{1}$ As we acknowledged in our introduction, marathon running results in a host of other physiological changes, some of which may lead to decrements in explicit memory. However, consideration of those factors alone provides no obvious explanation as to why marathon running led to improved implicit memory.

Healey, Campbell, and Hasher (2008) have reviewed task trade-offs that resemble the one shown here. Older adults, in their studies, showed impaired memory for material to which they were supposed to attend, yet showed enhanced memory for surrounding items that were supposed to have been ignored. The authors suggested that one effect of aging might be that people are less focused on the target task and may attend to information that is nonfocal to the nominal task. Priming of, and thus enhanced performance on, nonfocal material may ensue. This explanation holds up well for why older adults sometimes show memory enhancement, as compared with the performance of younger participants, depending on the task. However, in contrast to the results described with the older adults, in which attention was paid to items that were nonfocal at encoding, all of the materials in our experiment, both those used in the implicit task and those used in the explicit task, were focal at encoding. Participants made pleasantness judgments about all of the words, those that would later appear in the implicit condition and those later tested explicitly. Given that the words that would appear later in the implicit task and those that would appear in the explicit task were indistinguishable at encoding, a "dispersed attention" explanation does not seem able to explain the present results.

A simpler view of these effects might be that stress is just a distraction (Schmitter-Edgecombe, 1996), which might account for a finding of impaired performance on the explicit task. However, it cannot simultaneously account for enhanced performance on the implicit memory task. Simple distraction should hurt, not enhance, performance. We are left with the conclusion that stress enhanced some functions but impaired others.

These data contribute to our understanding of how stress mediates human memory. Interestingly, performance on the implicit memory task was enhanced, rather than unaffected or impaired. Amnesic patients typically show the same implicit memory performance as controls; they have not, in any published study, shown implicit memory performance that was significantly better than that of control participants. However, there are some salient differences between amnesic patients and marathon runners. The latter do not have organic brain damage, and it is highly unlikely that the stress hormones accompanying extreme exercise affect only one structure, such as the hippocampus (as is sometimes the case with amnesic patients). Stress hormones, including norepinephrine, endorphins, and others, are surging in marathon runners. It is not unreasonable to suppose that these hormones serve to improve certain kinds of mental function. Thus, either because the implicit and explicit systems have an inherently reciprocal relation in normals or, perhaps, because the different neuromodulators associated with extreme exercise have opposing effects on different subsystems, improving one while impairing another, the manipulation in our experiment revealed a double dissociation.

\section{AUTHOR NOTE}

We thank Shani Toledano, James Ware Billett, Toby Tanser, Ljubica Chatman, Leanne Zalewski, Nate Kornell, Robert A. Bjork, Edward E. Smith, and David H. Krantz. Address correspondence to T. S. Eich, Department of Psychology, Columbia University, 406B Schermerhorn Hall, Mail Code 5501, 1190 Amsterdam Avenue, New York, NY 10027 (e-mail: tse4@columbia.edu).

\section{REFERENCES}

Aloe, L., Bracci-laudiero, L., Alleva, L. E., Lambiase, A., Micera, A., \& Tirassa, P. (1994). Emotional stress induced by parachute jumping enhances blood nerve growth factor levels and the distribution of nerve growth factor receptors in lymphocytes. Proceedings of the National Academy of Sciences, 91, 10440-10444. doi:10.1073/pnas.91.22.10440

Angevaren, M., Aufdemkampe, G., Verhatr, H. J. J., Aleman, A., \& Vanhees, L. (2008). Physical activity and enhanced fitness to improve cognitive function in older people without known cognitive impairment. Cochrane Database of Systematic Reviews, 3, Art. No: CD005381. doi:10.1002/14651858.CD005381.pub3

Bäckman, L., Almkvist, O., Andersson, J., Nordberg, A., WinBLAD, B., REINECK, R., \& LÅNGSTROM, B. (1997). Brain activation in young and older adults during implicit and explicit retrieval. Journal of Cognitive Neuroscience, 9, 378-391. doi:10.1162/jocn.1997.9.3.378

Byrne, A. E., \& Byrne, D. G. (1993). The effect of exercise on depression, anxiety and other mood states: A review. Journal of Psychosomatic Research, 37, 565-574. doi:10.1016/0022-3999(93)90050-P

Cahill, L., \& Alkire, M. T. (2003). Epinephrine enhancement of human memory consolidation: Interaction with arousal at encoding. Neurobiology of Learning \& Memory, 79, 194-198. doi:10.1016/ S1074-7427(02)00036-9

Cook, N. J., Ng, A., Read, G. F., Harris, B., \& Riad-Fahmy, D. (1987). Salivary cortisol for monitoring adrenal activity during marathon runs. Hormone Research, 25, 18-23. doi:10.1159/000180628

de Kloet, E. R., Vreugdenhil, E., Oitzl, M. S., \& Jö̈Ls, M. (1998). 
Brain corticosteroid receptor balance in health and disease. Endocrine Reviews, 19, 269-301. doi:10.1210/er.19.3.269

Demers, L. M., Harrison, T. S., Halbert, D. R., \& Santen, R. J. (1981). Effect of prolonged exercise on plasma prostaglandin levels. Prostaglandins \& Medicine, 6, 413-418. doi:10.1016/0161 $-4630(81) 90073-2$

de Quervain, D. J.-F., Roozendaal, B., Nitsch, R. M., McGaugh, J. L., \& Hock, C. (2000). Acute cortisone administration impairs retrieval of long-term declarative memory in humans. Nature Neuroscience, 3, 313-314. doi:10.1038/73873

GRAF, P., \& MANDLER, G. (1984). Activation makes words more accessible, but not necessarily more retrievable. Journal of Verbal Learning \& Verbal Behavior, 23, 553-568. doi:10.1016/S0022-5371(84)90346-3

GraF, P., \& SCHACTER, D. L. (1985). Implicit and explicit memory for new associations in normal and amnesic subjects. Journal of Experimental Psychology: Learning, Memory, \& Cognition, 11, 501-518. doi:10.1037/0278-7393.11.3.501

Graf, P., \& Williams, D. (1987). Completion norms for 40 three-letter word stems. Behavior Research Methods, Instruments, \& Computers, 19, 442-445.

Healey, M. K., Campbell, K. L., \& Hasher, L. (2008). Cognitive aging and increased distractibility: Costs and potential benefits. In W. S. Sossin, J.-C. Lacaille, V. F. Castellucci, \& S. Belleville (Eds.), Essence of memory (Progress in Brain Research, Vol. 169, pp. 353363). Amsterdam: Elsevier. doi:10.1016/S0079-6123(07)00022-2

Kinlstrom, J. F., Schacter, D. L., Cork, R. L., Hurt, C. A., \& Behr, S. E. (1990). Implicit and explicit memory following surgical anesthesia. Psychological Science, 1, 303-306. doi:10.1111/j.1467-9280.1990 .tb00222.x

Kirschbaum, C., Pirke, K.-M., \& Hellhammer, D. H. (1993). The "Trier Social Stress Test": A tool for investigating psychobiological stress responses in a laboratory setting. Neuropsychobiology, 28, 7681. doi: $10.1159 / 000119004$

LeDoux, J. E. (1996). The emotional brain: The mysterious underpinnings of emotional life. New York: Simon \& Schuster.

McGAUGH, J. L. (2004). The amygdala modulates the consolidation of memories of emotionally arousing experiences. Annual Review of Neuroscience, 27, 1-28. doi:10.1146/annurev.neuro.27.070203.144157

Newcomer, J. W., Selke, G., Melson, A. K., Hershey, T., Craft, S.,
Richards, K., \& Alderson, A. L. (1999). Decreased memory performance in healthy humans induced by stress-level cortisol treatment. Archives of General Psychiatry, 56, 527-533. doi:10.1001/ archpsyc.56.6.527

Norbury, W. B., Herndon, D. N., Branski, L. K., Chinkes, D. L., \& JESCHKE, M. G. (2008). Urinary cortisol and catecholamine excretion after burn injury in children. Journal of Clinical Endocrinology \& Metabolism, 93, 1270-1275. doi:10.1210/jc.2006-2158

Pereira, A. C., Huddleston, D. E., Brickman, A. M., Sosunov, A. A., Hen, R., McKhann, G. M., Et AL. (2007). An in vivo correlate of exercise-induced neurogenesis in the adult dentate gyrus. Proceedings of the National Academy of Sciences, 104, 5638-5643. doi:10.1073/ pnas.0611721104

Resnick, H. S., Yehuda, R., Pitman, R. K., \& Foy, D. W. (1995). Effect of previous trauma on acute plasma cortisol level following rape. American Journal of Psychiatry, 152, 1675-1677.

SAPOLSKY, R. M. (2004). Why zebras don't get ulcers: An updated guide to stress, stress-related diseases, and coping (3rd ed.). New York: Holt.

Schmitter-Edgecombe, M. (1996). The effects of divided attention on implicit and explicit memory performance. Journal of the International Neuropsychological Society, 2, 111-125. doi:10.1037/0894 $-4105.10 .2 .155$

Taylor, M. K., Sausen, K. P., Potterat, E. G., Mujica-Parodi, L. R., ReIs, J. P., MARKhaM, A. E., ET AL. (2007). Stressful military training: Endocrine reactivity, performance, and psychological impact. Aviation, Space, \& Environmental Medicine, 78, 1143-1149. doi:10.3357/ ASEM.2151.2007

Uchakin, P. N., Gotovtseva, E. P., \& Stray-Gundersen, J. (2003). Immune and neuroendocrine alterations in marathon runners. Journal of Applied Research in Clinical \& Experimental Therapeutics, 3, 483-494.

\section{NOTE}

1. An anonymous reviewer suggested that the Yerkes-Dodson law might be applicable to these results.

(Manuscript received December 9, 2008; revision accepted for publication February 13, 2009.) 